\title{
Carbon Dots: From Lab Synthesis to Unique Applications ${ }^{\dagger}$
}

\author{
Yiqun Zhou ${ }^{1, *}$, Roger M. Leblanc ${ }^{1}$ \\ 1 Department of Chemistry, University of Miami, USA \\ * Correspondence: yxz431@ miami.edu \\ $\dagger$ Presented at Materials Chemistry and Physics (Materials Chemistry 2020) - International e-Conference
}

Received: 16.09.2020; Revised: 20.09.2020; Accepted: 24.09.2020; Published: 27.09.2020

\begin{abstract}
Carbon dots (CDs) are a group of relatively new carbon-based spherical nanoparticles (NPs) with diameters less than $10 \mathrm{~nm}$. They are widely present in nature and can also be synthesized using various carbon-based substances as precursors by either top-down or bottom-up approaches. They are well characterized for tunable surface functionality, excellent photoluminescence (PL), high photostability and water dispersity, good biocompatibility, and nontoxicity. Also, they display different sizes and surface chemistry depending on the preparation methods, and precursors applied. Applications of CDs in drug delivery, bioimaging, sensing, optics, photocatalysis, and other nanotechnology fields are rapidly rising due to their aforementioned unique properties.

In this presentation, I will focus on the preparation, characterization and several significant applications of the CDs developed in our group. Specifically, I will introduce one type of CDs in yellow powder (Y$\mathrm{CDs}$ ) together with their amphiphilicity, tunable PL, and nontoxicity. In addition, Y-CDs showed an excellent permeability of body tissues, even including the blood-brain barrier (BBB) via passive diffusion using a zebrafish model. Furthermore, Y-CDs exhibited a strong inhibitory effect on the secretion of amyloid precursor protein (APP) and beta-amyloid (A $\beta$ ), which is significant for the future treatment of Alzheimer's disease (AD). Considering their excellent permeability, Y-CDs are also promising drug nanocarriers for the treatment of diverse viral diseases such as COVID-19. Another work I would like to share with the public is that CDs have proved to be promising Lego-like building blocks for the assembly of novel versatile drug nanocarriers to simultaneously fulfill multitasks.
\end{abstract}

Keywords: carbon dots; drug nanocarrier; zebrafish model; blood-brain barrier penetration; Alzheimer's disease treatment; COVID-19 treatment; nanoparticle assembly.

\footnotetext{
(C) 2020 by the authors. This article is an open-access article distributed under the terms and conditions of the Creative Commons Attribution (CC BY) license (https://creativecommons.org/licenses/by/4.0/).
}

\section{Funding}

This research was funded by the National Science Foundation, grant number 1809060 and 2041413.

\section{Acknowledgments}

This research has no acknowledgment. 


\section{Conflicts of Interest}

The authors declare no conflict of interest.The funders had no role in the design of the study; in the collection, analyses, or interpretation of data; in the writing of the manuscript, or in the decision to publish the results. 